\title{
Assistência de enfermagem obstétrica baseada em boas práticas: do acolhimento ao parto
}

\author{
Maraysa Jéssyca de Oliveira Vieira ${ }^{1}$, Amuzza Aylla Pereira dos Santos ${ }^{2}$, \\ Jovânia Marques de Oliveira e Silva ${ }^{3}$, Maria Elisângela Torres de Lima Sanches ${ }^{4}$
}

\footnotetext{
${ }^{1}$ Discente do curso de graduação em Enfermagem da Escola de Enfermagem e Farmácia da Universidade Federal de Alagoas. Maceió, AL, Brasil. E-mail: maraysa jessyca@hotmail.com. ${ }^{2}$ Enfermeira, Mestre em Ciências da Saúde. Discente do Programa de Pós-Graduação em Ciências da Saúde, nível Doutorado, da Universidade Federal de Alagoas. Maceió, AL, Brasil. E-mail: amuzzasantos@bol.com.br.

${ }^{3}$ Enfermeira, Doutora em Enfermagem. Professora Adjunto da Escola de Enfermagem e Farmácia da Universidade Federal de Alagoas. Maceió, AL, Brasil. E-mail: jovaniasilva@gmail.com.

${ }^{4}$ Enfermeira, Mestre em Enfermagem. Professor Auxiliar da Escola de Enfermagem e Farmácia da Universidade Federal de Alagoas. Maceió, AL, Brasil. E-mail: sanches_eli@hotmail.com.
}

Recebido: 14/07/2015.

Aceito: 23/03/2016.

Publicado: 30/06/2016.

Como citar esse artigo:

Vieira MJO, Santos AAP, Silva JMO, Sanches METL. Assistência de enfermagem obstétrica baseada em boas práticas: do acolhimento ao parto. Rev. Eletr. Enf. [Internet]. 2016 [acesso em:_____;18:e1166. Disponível em http://dx.doi.org/10.5216/ree.v18.36714.

\section{RESUMO}

Objetivou-se avaliar a assistência do enfermeiro obstetra do acolhimento ao parto, baseando-se nas boas práticas obstétricas. Trata-se de um estudo descritivo, retrospectivo e documental, de abordagem quantitativa por meio da análise de 500 prontuários. A pesquisa identificou que houve a realização da episiotomia em 12,2\%, amniotomia em $13 \%$, o uso de ocitocina em $42,8 \%$, os métodos não farmacológicos para alívio da dor foram aplicados em 75,4\% e o contato pele a pele foi estimulado em $91,6 \%$. Verificou-se que o uso das boas práticas obstétricas recomendadas pela Organização Mundial da Saúde foram utilizadas pelo enfermeiro obstetra na assistência do acolhimento ao parto na sua maioria e está próxima do preconizado pelas evidências científicas.

Descritores: Cuidados de Enfermagem; Enfermagem Obstétrica; Assistência Perinatal; Acolhimento.

\section{INTRODUÇÃO}

Na história da saúde pública, a atenção materno-infantil tem sido considerada uma área prioritária, principalmente no que se refere aos cuidados da mulher durante a gestação, a fim de manter um ciclo gravídico-puerperal com menor risco possível para o trinômio mãe-filho-família(1).

A gestação é vivenciada de maneira especial na vida da mulher, cada uma com suas vivências transformam o parto em um processo cultural, pois reflete os valores históricos e sociais prevalentes em cada sociedade. O modelo atual de atenção ao parto e nascimento no Brasil traz como caracterização o excesso de intervenção tirando da mulher o poder de condução do mesmo, o que tem contribuído 
significativamente para o aumento de taxas de partos cesáreos e a morbimortalidade materna e perinatal ${ }^{(2-}$ 3).

Nessa perspectiva as evidências científicas utilizadas através das boas práticas obstétricas ajudaram na condução para melhorar os fatores da saúde materna que atuam negativamente durante o ciclo gestacional, influenciando diretamente os resultados na assistência do pré-natal de qualidade contribuindo assim para a redução de danos no trinômio. Da mesma forma, uma parcela importante das complicações que podem ocorrer ao longo do trabalho de parto e no momento do parto foi reduzida por cuidado obstétrico apropriado, realizado com o uso adequado de tecnologia. Estas várias práticas utilizadas na assistência à gestação e ao parto vem sendo promotoras das experiências exitosas nos processos obstétricos e são efetivadas pela redução de desfechos perinatais negativos ${ }^{(4-5)}$.

Seguindo as recomendações da Organização Mundial de Saúde (OMS) e as do Ministério da Saúde (MS) as instituições vêm incentivando a realização do parto normal e a diminuição das cesarianas. Essas medidas de humanização visam proporcionar bem-estar à mulher e reduzir riscos para ela e seu bebê, como também proporcionar conforto e bem-estar ao acompanhante ${ }^{(6)}$.

Dessa forma foi estabelecido um compromisso fundamental que os profissionais de saúde envolvidos na atenção à saúde da mulher possam assisti-la no momento do parto e nascimento com segurança e dignidade, atendendo as recomendações preconizadas pelo MS, pois humanizar o parto é promover assistência de qualidade à parturiente utilizando as classificações das boas práticas obstétricas que estão divididas em quatro categorias:

- Categoria A - Práticas demonstradamente úteis e que devem ser estimuladas;

- Categoria B - Práticas claramente prejudiciais ou ineficazes e que devem ser eliminadas; Categoria C - Práticas em relação às quais não existem evidências suficientes para apoiar uma recomendação clara e que devem ser utilizadas com cautela, até que mais pesquisas esclareçam a questão;

- Categoria D - Práticas frequentemente utilizadas de modo inadequado ${ }^{(7)}$.

Essas categorias foram criadas para fortalecer a utilização correta das boas práticas e promover uma assistência adequada no momento do trabalho de parto e parto, visando uma assistência baseada no alívio da dor, conforto físico e emocional, liberdade de escolha para o nascimento do seu bebê e a melhor via de parto, dando suporte (material, pessoal e emocional) necessário para o trinômio e ajudar a mulher a vivenciar o processo de parturição de forma mais segura, tranquila, satisfatória e feliz ${ }^{(7-8)}$.

Além disso, ao prestar uma assistência de qualidade à mulher que vivencia o ciclo gravídico puerperal, os profissionais podem ajudá-la a superar medos, tensões e ansiedades, por meio do exercício da empatia e respeito, considerando as opiniões, preferências e suas necessidades ${ }^{(9)}$.

Os profissionais de saúde são coadjuvantes dessas experiências, desempenhando um papel satisfatório ao colocar seu conhecimento a serviço do bem-estar da mulher e do bebê, ajudando-os no processo de parturição e nascimento de forma saudável ${ }^{(6)}$.

Nesse contexto, o enfermeiro obstetra exerce papel imprescindível na atenção durante o parto e o 
nascimento e sua atuação vem sendo requerida, tanto nos cenários de cuidado que envolve ações de prénatal, parto e puerpério, quanto na formulação e desenvolvimento de políticas relacionadas com o contexto obstétrico $^{(6,10)}$. É no momento do parto e nascimento que o enfermeiro obstetra pode atuar de maneira determinante neste processo, fazendo o diferencial do cuidado prestado, na capacidade de apoio e comunicação entre os sujeitos envolvidos pela ação do trabalho de parto e parto, favorecendo assim o contato e o vínculo, pois a gestação, o parto e puerpério constituem experiências significativas e enriquecedoras para todos os que delas participam ${ }^{(11-12)}$.

Por esses motivos a relevância deste estudo caracteriza-se por ser a assistência ao parto e nascimento uma temática de discussão internacional, onde a utilização das boas práticas obstétricas pelos profissionais de saúde e dentre eles o enfermeiro obstetra tem empreendido esforços no sentido de reduzir os danos ao ciclo gravídico puerperal diminuindo assim os altos índices de cesariana e de mortalidade materna e neonatal, além de melhorar a qualidade da assistência ao trinômio ${ }^{(6,13)}$.

Assim, diante da importância da temática objetivou-se avaliar a assistência do enfermeiro obstetra do acolhimento ao parto, baseando-se nas boas práticas obstétricas.

\section{MÉTODO}

Estudo descritivo, retrospectivo e documental, de abordagem quantitativa. A pesquisa foi realizada em uma Maternidade de Risco Habitual em uma capital do nordeste brasileiro. O tamanho da amostra foi estimado em 462 prontuários de mulheres atendidas considerando a proporção na população de 50\% (estimativa mais segura quando se tem pouca informação da população estudada), a precisão absoluta de $6 \%$, e o nível de significância de 1\%. Foi usada calculadora eletrônica disponível na internet no URL: http://www.lee.dante.br/pesquisa/amostragem/calculo_amostra.html.

Para a composição da amostra, foi realizado um levantamento documental arquivado, por meio de prontuários, fichas de atendimento obstétrico e livros de registro, utilizados durante o acolhimento da gestante até o parto, no ano de 2014. No total foram analisados 500 prontuários, de janeiro a junho de 2015 com uma média de 50 prontuários por mês.

Para a coleta de dados foi elaborado um formulário semiestruturado com dados de identificação para caracterizar a amostra e dados específicos sobre o atendimento realizado por enfermeiras obstetras às parturientes. Os dados foram coletados após aprovação do projeto pelo Comitê de Ética e Pesquisa da Universidade Federal de Alagoas, sob o processo no: 40166414.8.0000.5013, no dia 16 de janeiro de 2015.

As variáveis utilizadas no estudo foram: preenchimento do formulário de coleta de dados, idade, procedência, cor/raça, estado civil, escolaridade, gestações e paridade da paciente, quantidade de consultas de pré-natal, utilização do partograma, utilização da episiotomia, manobras ativas no terceiro estágio de parto, realização da amniotomia, uso de ocitocina, contato pele a pele, métodos não farmacológicos para alívio da dor, local do parto e idade gestacional.

Para a organização, tabulação e análise dos dados estatísticos descritivos utilizou-se o programa 
Microsoft Excel 2010. A análise descritiva ocorreu a partir de frequência absoluta ( $n$ ) e percentual (F\%) e os resultados foram apresentados de forma descritiva, por meio de tabelas.

\section{RESULTADOS}

Dos 500 (100\%) prontuários analisados, $413(83,0 \%)$ estavam com o preenchimento completo e 87 $(17,0 \%)$ estavam parcialmente preenchidos, ou seja, faltavam dados essenciais de identificação da parturiente, do acompanhamento da evolução do trabalho de parto e parto e do pós-parto.

A Tabela 1 refere-se ao perfil das mulheres atendidas por enfermeiras obstetras nesse período na maternidade.

Tabela 1: Perfil sociodemográfico das parturientes numa Maternidade de baixo risco. Maceió, AL, Brasil, 2014.

\begin{tabular}{|c|c|c|}
\hline Variável & (n) & (F\%) \\
\hline \multicolumn{3}{|l|}{ Faixa Etária } \\
\hline Menor ou igual a 15 anos & 32 & 6,4 \\
\hline 16 a 25 anos & 330 & 66 \\
\hline 26 a 35 anos & 138 & 27,6 \\
\hline \multicolumn{3}{|l|}{ Município de procedência } \\
\hline Maceió & 228 & 45,6 \\
\hline Atalaia & 39 & 7,8 \\
\hline Marechal Deodoro & 36 & 7,2 \\
\hline Rio Largo & 30 & 6 \\
\hline São Luís do Quitunde & 27 & 5,4 \\
\hline \multicolumn{3}{|l|}{ Cor } \\
\hline Branca & 35 & 7 \\
\hline Preta & 26 & 5,2 \\
\hline Parda & 416 & 83,2 \\
\hline \multicolumn{3}{|l|}{ Estado civil } \\
\hline Solteira & 404 & 80,8 \\
\hline Casada & 79 & 15,8 \\
\hline \multicolumn{3}{|l|}{ Escolaridade } \\
\hline Ensino fundamental incompleto & 189 & 37,8 \\
\hline Ensino fundamental completo & 79 & 15,8 \\
\hline Ensino médio incompleto & 58 & 11,6 \\
\hline Ensino médio completo & 89 & 17,8 \\
\hline
\end{tabular}

Quanto aos dados obstétricos das pacientes, a parcela maior era de primigesta e primípara. Já em relação à realização de consultas durante o pré-natal da última gestação, mais da metade $(62,0 \%)$ das mulheres realizaram mais de seis consultas. Ainda em relação à gestação atual, quase a totalidade $(95,8 \%)$ das mulheres chegaram à maternidade para a realização do parto com idade gestacional de 37 a 42 semanas, conforme descrito na Tabela 2.

Tabela 2: Perfil obstétrico das parturientes numa Maternidade de baixo risco. Maceió, AL, Brasil, 2014.

\begin{tabular}{llll}
\hline & Variável & (n) & (F\%) \\
\hline Gestações & & & \\
Primigesta & & 207 & 41,4
\end{tabular}




\begin{tabular}{|c|c|c|}
\hline Secundigesta & 136 & 27,2 \\
\hline Tercigesta & 73 & 14,6 \\
\hline Multigesta & 82 & 16,4 \\
\hline Não registrado & 2 & 0,4 \\
\hline Total & 500 & 100 \\
\hline \multicolumn{3}{|l|}{ Paridade } \\
\hline Primípara & 224 & 44,8 \\
\hline Paucípara (Até 3 partos) & 209 & 41,8 \\
\hline Multípara & 65 & 13 \\
\hline Não registrado & 2 & 0,4 \\
\hline Total & 500 & 100 \\
\hline \multicolumn{3}{|l|}{ Consultas de pré-natal } \\
\hline Nenhuma & 4 & 0,8 \\
\hline 1 a 3 & 30 & 6 \\
\hline 4 a 6 & 101 & 17,8 \\
\hline Mais de 6 & 298 & 62 \\
\hline Não registrado & 67 & 13,4 \\
\hline Total & 500 & 100 \\
\hline \multicolumn{3}{|l|}{ Idade gestacional atual } \\
\hline Pré-termo & 13 & 2,6 \\
\hline Termo & 477 & 95,4 \\
\hline Pós-termo & 2 & 0,4 \\
\hline Não registrado & 8 & 1,6 \\
\hline Total & 500 & 100 \\
\hline
\end{tabular}

No que concerne as boas práticas realizadas pela assistência de enfermagem obstétrica às parturientes da maternidade em questão, foram analisados os dados referentes a utilização do partograma e da episiotomia, as manobras ativas do terceiro estágio, a realização da amniotomia, o uso de ocitocina, contato pele a pele com o bebê após o nascimento, a utilização de métodos não farmacológicos para alívio da dor e o local do parto.

A utilização do partograma para registro da evolução do trabalho de parto foi encontrada em 379 $(75,8 \%)$ prontuários, onde $182(48,0 \%)$ estavam completamente preenchidos, 65 (17,0\%) com preenchimento incompleto e $132(35,0 \%)$ sem preenchimento. Quanto à utilização da episiotomia, esta foi realizada em apenas $61(12,2 \%)$ partos.

As manobras ativas no terceiro estágio de parto foram realizadas em $489(97,8 \%)$ partos, entretanto as três manobras (Ocitocina intramuscular, tração controlada do cordão e massagem uterina) preconizadas foram realizadas juntas em apenas dois partos. Quanto à realização da amniotomia, esta foi realizada em 66 $(13,2 \%)$ partos, entretanto, desses, $20(4,0 \%)$ foram realizados pelo profissional médico. Em referência ao uso de ocitocina no trabalho de parto, a taxa de utilização foi relativamente alta, evidenciado em 214 (42,8\%) parturientes.

Já os métodos não farmacológicos para alívio da dor, estes foram utilizados em 377 (75,4\%) parturientes, entretanto 197 (39,4\%) constavam nos prontuários quais eram esses métodos, e 180 (36\%) constavam no livro de registro de parto normal que os métodos foram aplicados, mas no prontuário não havia registro. Entre os métodos utilizados, os que mais tiveram destaque foi a bola suíça utilizada em 100 
(20,0\%) parturientes, deambulação realizada por 94 mulheres $(18,8 \%)$ e o banho de aspersão aplicado em $61(12,2 \%)$ parturientes.

No que diz respeito ao local de realização do parto, 487 (97,4\%) deles aconteceram na sala de parto e os demais no pré-parto. Quanto ao contato pele a pele do binômio mãe-filho logo após o nascimento, este aconteceu em $458(91,6 \%)$ partos.

\section{DISCUSSÃO}

Neste estudo, analisando a assistência prestada pelo enfermeiro obstetra mediante o uso das boas práticas obstétricas e classificando através das categorias, evidenciou-se que uso do partograma para registro da evolução do trabalho de parto apresentou preenchimento completo em menos da metade demonstrando a pouca importância dada à utilização desse instrumento para acompanhamento do trabalho de parto.

O partograma é uma tecnologia utilizada na área da obstetrícia que auxilia na atenção e acompanhamento da evolução do trabalho de parto. A OMS, no ano de 1994, recomendou o seu uso durante o trabalho de parto, com o objetivo de reduzir a morbidade e mortalidade materna e fetal. Dessa forma, o MS preconiza a adoção dos indicadores de qualidade no período gravídico-puerperal, em que se insere o partograma como forma de acompanhar e avaliar o processo de parturição ${ }^{(14-15)}$. O monitoramento cuidadoso do progresso do parto através do partograma é classificado dentro das boas práticas relacionadas à atenção ao parto como categoria $\mathrm{A}$, ou seja, como prática útil e que deve ser estimulada, pois o seu uso incorreto ou incompleto pode modificar o processo do trabalho de parto e parto de forma negativa provocando assim mortes desnecessárias ${ }^{(8)}$.

$\mathrm{Na}$ assistência obstétrica o enfermeiro obstetra deve compreender a importância do uso sistemático e possuir habilidade na utilização e preenchimento do partograma, pois, é necessário para os profissionais que pretendem ter uma atuação competente, humanizada, segura e assertiva à parturição. Todavia, essa tecnologia ainda é pouco explorada no cotidiano da prática do enfermeiro obstetra ${ }^{(9)}$.

A episiotomia foi pouco utilizada nos partos com uma taxa de 12,2\%, bem próximo do recomendado pela OMS, demostrando vantagens para as parturientes, considerando, dessa forma, uma assistência adequada, refletindo a diminuição de um procedimento invasivo desnecessário a evolução do parto. A episiotomia é um procedimento cirúrgico usado em obstetrícia para aumentar a abertura vaginal através de uma incisão no períneo ao final do segundo estágio do parto vaginal, porém quando o períneo é bem trabalhado utilizando-se a posição adequada e desejada pela mulher esta intervenção cirúrgica torna-se desnecessária ${ }^{(10)}$.

Embora a episiotomia tenha se tornado o procedimento cirúrgico mais comum do mundo, foi introduzida sem muita evidência científica sobre sua real efetividade. Por isso, atualmente em nível mundial, há uma intenção de torná-la um procedimento restrito e não mais rotineiro ${ }^{(11)}$.

A recomendação atual da OMS não é de proibir a episiotomia, mas de restringir seu uso, até porque, 
em alguns casos, pode ser necessário, como em situações de sofrimento fetal, progresso insuficiente do parto e lesão iminente de 3 o grau do períneo. A OMS sugere ainda que a taxa ideal de episiotomia nos diversos serviços seja em torno de $10 \%$, realidade em muitos países europeus ${ }^{(13)}$. A prática liberal e rotineira de episiotomia durante o parto é classificada como categoria B, ou seja, claramente prejudicial ou ineficaz e que deve ser eliminada ${ }^{(14)}$.

Nesse estudo identificou-se ainda que a maioria utilizou as manobras ativas no terceiro estágio de parto (Categoria A) , seguindo recomendações do MS. O terceiro período do parto é o período que se inicia com o nascimento até a saída da placenta (delivramento). É classificado como prolongado se não se completar com 30 minutos, quando se adota conduta ativa, e 60 minutos com conduta fisiológica. Complicações no terceiro período do parto são importantes causas de mortalidade materna por todo o mundo ${ }^{(15)}$.

$\mathrm{Na}$ maternidade em estudo foi evidenciada uma taxa relativamente baixa de amniotomia. A pouca realização desse procedimento reflete em vantagens tanto para parturiente como para o bebê. De acordo com a OMS a amniotomia precoce de rotina para diminuir a duração do trabalho de parto também é classificada como categoria $\mathrm{B}^{(16)}$.

Embora existam evidências de que a amniotomia precoce possa diminuir a duração do trabalho de parto, efeitos indesejáveis podem ocorrer, como o incremento de desacelerações precoces dos batimentos cardíacos fetais e alterações sobre o polo cefálico. Além dessas complicações, quanto maior a duração do parto com membranas rotas, maior o risco de infecção ovular e puerperal. Assim, a rotura artificial da bolsa deve ser evitada, restringindo seu uso para aquelas condições onde sua prática seja claramente benéfica, como é o caso de algumas distorcias funcionais ${ }^{(17)}$.

Quanto ao uso de ocitocina no trabalho de parto, foi identificada uma taxa de utilização alta, evidenciado em $42,8 \%$ das parturientes, muitas vezes fazendo com que as parturientes sintam dores desnecessárias. Não existem benefícios comprovados no uso rotineiro de ocitocina, mas existem efeitos colaterais, tais como a hiperestimulação uterina e o aumento da dor ${ }^{(17)}$. A OMS não recomenda infusão rotineira de ocitocina em parturientes saudáveis. $\mathrm{O}$ uso de ocitocina é desnecessário e pode ser prejudicial. A administração de ocitócitos em qualquer momento antes do parto de um modo que não permita controlar seus efeitos deve ser eliminada da assistência, esta prática foi classificada como categoria $\mathrm{B}^{(16)}$.

Em relação aos métodos não farmacológicos para alívio da dor, a maior parte das mulheres atendidas fizeram uso, e os que mais se destacaram foram a bola suíça, deambulação e o banho de aspersão. $\mathrm{O}$ uso de métodos não farmacológicos de alívio da dor, além de proporcionar conforto à parturiente, evita o uso de substâncias farmacológicas que possam interferir no processo fisiológico do parto ${ }^{(17)}$. Eles diminuem a dor provocada pelas contrações uterinas, aumentam a satisfação materna e melhoram os resultados obstétricos de modo que as mulheres se apresentam mais colaborativas, pois apreciam a sensação de controle que ganham ao manejarem ativamente a dor e o apoio que recebem do acompanhante e dos cuidadores, além da liberdade de movimentação e de escolha dos movimentos. Esta prática está inserida na categoria $\mathrm{A}^{(18)}$. 
No que diz respeito ao local de realização do parto, a maioria aconteceu na sala de parto. Entretanto, independente do local do parto, o que importa é que haja empoderamento da mulher, respeitando sua fisiologia e desejos, a fim de oferecer uma assistência humanizada ${ }^{(17)}$.

Com um resultado bastante estimulante, o contato pele a pele do binômio mãe-filho logo após o nascimento aconteceu na quase totalidade dos partos. $\mathrm{O}$ contato pele a pele deve iniciar imediatamente após o nascimento, ser contínuo, prolongado e estabelecido entre todo binômio saudáveis. O contato pele a pele acalma o bebê e a mãe que entram em sintonia única proporcionada por esse momento. Além disso, auxilia na estabilização sanguínea, dos batimentos cardíacos e respiratórios da criança; reduz o choro e o estresse do recém-nascido com menor perda de energia e mantém o bebê aquecido pela transmissão de calor de sua mãe ${ }^{(18)}$.

A prática de incentivo ao contato precoce e a amamentação e tempo entre o nascimento e a mamada, revelam-se como dados que estão de acordo com as recomendações do MS, que preconiza o contato da mãe com seu bebê e aleitamento materno na sala de parto. O recém-nascido sobre o peito da mãe, imediatamente após o parto, proporciona um ambiente ótimo para sua adaptação à vida extrauterina e para a adaptação da mulher à maternidade ${ }^{(19-20)}$.

O enfermeiro obstetra possui um papel importante nesse momento da assistência, pois as orientações e o incentivo ao contato precoce podem favorecer o vínculo entre mãe e filho, além de ser uma ação que potencializa a promoção do aleitamento materno ${ }^{(17)}$.

O estudo evidenciou ainda que as boas práticas obstétricas foram realizadas de maneira significativa como preconizam a OMS e o MS, com destaque da atuação do enfermeiro obstetra na promoção da humanização do parto e nascimento para melhor qualidade de vida e saúde do binômio mãe e filho(19).

\section{CONCLUSÃO}

O resultado obtido com a avaliação das variáveis como a taxa de episiotomia, os métodos não farmacológicos para alívio da dor, o contato pele a pele mãe-bebê, favorecendo o quanto antes a formação do vínculo entre esse binômio, a pouca realização de amniotomia que permite identificar o compromisso do enfermeiro obstetra com a promoção da saúde na qualidade da assistências ao trinômio, demonstrando assim experiências exitosas no processo de trabalho de parto e parto mediante o uso das boas práticas obstétricas.

Demonstra também que o investimento na utilização de boas práticas obstetras têm sido extremamente válidas, principalmente considerando os importantes benefícios proporcionados ao trinômio, além de empoderar a mulher no seu processo de parturição. Assim, fica clara a importância do enfermeiro obstetra prestar uma assistência integral e com competência técnica e científica, capaz de mensurar as necessidades humanas, para se utilizar as condutas úteis e recomendadas pela OMS no trabalho de parto e parto, em maternidade de risco habitual.

Essa pesquisa aponta reflexão sobre a qualidade da assistência de enfermagem obstétrica, subsidiada 
por evidencias cientificas tanto para aplicação das boas práticas quanto a utilização de intervenções quando necessárias.

\section{REFERÊNCIAS}

1. Firmo WCA, Paredes AO, Almeida AC, Campos MC, Pimentel MIC, Pontes SRS. Perfil dos exames laboratoriais em gestantes atendidas no Centro de Saúde de Lago Verde, Maranhão, Brasil. J Manag Prim Heal Care [Internet]. 2013 [acesso em: 30 jun. 2016];4(2):77-86. Disponível em: http://www.jmphc.com.br/saude-

publica/index.php/jmphc/article/view/173.

2. Hodnett ED, Downe S, Walsh D. Alternative versus conventional institutional settings for birth. Cochrane database Syst Rev [Internet]. 2012 [acesso em: 30 jun. 2016];(8):CD000012. Disponível em:

http://dx.doi.org/10.1002/14651858.CD000012.pub4.

3. Bergstrom M. Continuous support in labour has beneficial effects for mother and baby. Evid Based Med [Internet]. 2011 [acesso em: 30 jun. 2016];16(6):182-3. Disponível em: http://dx.doi.org/10.1136/ebm1409.

4. Velasque EAG, Cabral FB, Pradebon VM. O enfermeiro no processo parir/nascer: estratégia de cuidado e humanização do parto. Rev Enferm da UFSM [Internet]. 2011 [acesso em: 30 jun. 2016];1(1):80-7. Disponível em: http://periodicos.ufsm.br/reufsm/article/view/1991.

5. Lobo SF, Oliveira SMJV, Schneck CA, Silva FMB, Bonadio IC, Riesco MLG. Resultados maternos e neonatais em Centro de Parto Normal peri-hospitalar na cidade de São Paulo, Brasil. Rev Esc Enferm USP [Internet]. 2010 [acesso em: 30 jun. 2016];44(3):812-8. Disponível em: http://dx.doi.org/10.1590/S0080-62342010000300037.

6. Jamas MT, Hoga LA, Tanaka AC. Mothers' birth care experiences in a Brazilian birth centre. Midwifery [Internet]. 2011 [acesso em: 30 jun. 2016];27(5):693-9. Disponível em: http://dx.doi.org/10.1016/j.midw.2009.10.004.

7. Ministério da Saúde. Humanização do parto e do nascimento (Cadernos HumanizaSUS; v. 4) [Internet]. Brasília: Ministério da Saúde, 2014 [acesso em: 30 jun. 2016]. Disponível em:

http://www.redehumanizasus.net/sites/default/files/caderno_humanizasus_v4_humanizacao_parto.pdf.

8. Porfírio AB, Progianti JM, Souza DOM. As práticas humanizadas desenvolvidas por enfermeiras obstétricas na assistência ao parto hospitalar. Rev. Eletr. Enf. [Internet]. 2010 [acesso em: 30 jun. 2016];12(2):331-6. Disponível em: http://dx.doi.org/10.5216/ree.v12i2.7087.

9. Gomes ML, Moura MAV, Souza IEO. Obstetrical practice by nurses in instiutional childbirth: a possibility for emancipatory knowledge. Texto contexto - enferm. [Internet]. 2013 [acesso em: 30 jun. 2016];22(3):763-71. Disponível em: http://dx.doi.org/10.1590/S0104-07072013000300024.

10. Reis JTS, Saraiva FO, Ferraresi MF, Vieira MAS. Perfil epidemiológico das parturientes atendidas em uma maternidade de alto risco de Goiânia-GO. Estudos [Internet]. 2014 [acesso em: 30 jun. 2016];41(2):329-39. Disponível em: http://seer.ucg.br/index.php/estudos/article/view/3388.

11. Vasconcelos KL, Martins CA, Mattos DV, Tyrrell MAR, Bezerra ALQ, Porto J. Partograma: instrumento para segurança na assistência obstétrica. Rev enferm UFPE online [Internet]. 2013 [acesso em: 30 jun. 2016];7(2):619-24. Disponível em: http://www.revista.ufpe.br/revistaenfermagem/index.php/revista/article/view/3717.

12. Salge AKM, Lôbo SF, Siqueira KM, Silva RCR, Guimarães JV. Prática da episiotomia e fatores maternos e neonatais relacionados. Rev. Eletr. Enf. [Internet]. 2012 [acesso em: 30 jun. 2016];14(4):779-85. Disponível em:

http://dx.doi.org/10.5216/ree.v14i4.17538.

13. Amorim MMR, Port AMF, Souza ASR. Assistência ao segundo e terceiro períodos do trabalho de parto baseada em evidências. Femina [Internet]. 2010 [acesso em: 30 jun. 2016];38(11):583-91. Disponível em:

http://www.febrasgo.org.br/site/wp-content/uploads/2013/05/Feminav38n11_583-591.pdf.

14. Dahlen HG, Tracy S, Tracy M, Bisits A, Brown C, Thornton C. Rates of obstetric intervention among low-risk women giving birth in private and public hospitals in NSW: a population-based descriptive study. BMJ Open [Internet]. 2012 [acesso em: 30 jun. 2016];2(5):e001723. Disponível em: http://dx.doi.org/10.1136/bmjopen-2012-001723.

15. Busanello J, Kerber NPC, Mendoza-Sassi RA, Mano PS, Susin LRO, Gonçalves BG. Atenção humanizada ao parto de adolescentes: análise das práticas desenvolvidas em um centro obstétrico. Rev Bras Enferm [Internet]. 2011 [acesso em: 30 jun. 2016];64(5):824-32. Disponível em: http://dx.doi.org/10.1590/s0034-71672011000500004.

16. Cruz LMN, Cruz AGC. A utilização da Bola Suíça na promoção do parto humanizado. Rev Bras Ciências da Saúde [Internet]. 2015 [acesso em: 30 jun. 2016];18(2):175-80. Disponível em: 
http://periodicos.ufpb.br/ojs/index.php/rbcs/article/view/16698.

17. Gayeski ME, Brüggemann OM. Métodos não farmacológicos para alívio da dor no trabalho de parto: uma revisão sistemática. Texto contexto - enferm [Internet]. 2010 [acesso em: 30 jun. 2016];19(4):774-82. Disponível em: http://dx.doi.org/10.1590/S0104-07072010000400022.

18. Matos TA, Souza MS, Santos EKA, Velho MB, Seibert ERC, Martins NM. Contato precoce pele a pele entre mãe e filho: significado para mães e contribuições para a enfermagem. Rev Bras Enferm [Internet]. 2010 [acesso em: 30 jun. 2016];63(6):998-1004. Disponível em: http://dx.doi.org/10.1590/S0034-71672010000600020.

19. Gomes ARM, Pontes DS, Pereira CCA, Brasil AOM, Moraes LCA. Assistência de enfermagem obstétrica na humanização do parto normal. Rev Recien - Rev Científica Enferm [Internet]. 2014 [acesso em: 30 jun. 2016];(11):23-7. Disponível em: http://www.recien.com.br/online/index.php/Recien/article/view/73.

20. Victora CG, Aquino EM, Leal MC, Monteiro CA, Barros FC, Szwarcwald CL. Maternal and child health in Brazil: progress and challenges. Lancet [Internet]. 2011 [acesso em: 30 jun. 2016];377(9780):1863-76. Disponível em: http://dx.doi.org/10.1016/S0140-6736(11)60138-4. 